\title{
A short proof of Morley's theorem
}

Yoshitake Hashimoto

Yoshitake Hashimoto received his D.Sc. from University of Tokyo in 1990. He then had a postdoctoral position at University of Tokyo. Since 1994 he has a position at Osaka City University, where he is now associate professor in the Department of Mathematics. His main fields of research are topology and differential geometry.

We present a proof of the following:

Morley's theorem (1899) In any triangle, the three points of intersection of the adjacent angle trisectors form an equilateral triangle.

Proof. Let $\alpha, \beta, \gamma$ be arbitrary positive angles with $\alpha+\beta+\gamma=60^{\circ}$. For any angle $\eta$ we put $\eta^{\prime}:=\eta+60^{\circ}$.

Let $\triangle D E F$ be an equilateral triangle, and $A$ [resp. $B, C]$ be the point lying opposite to $D$ [resp. $E, F]$ with respect to $E F$ [resp. $F D, D E$ ] and satisfying $\angle A F E=\beta^{\prime}$, $\angle A E F=\gamma^{\prime}$ [resp. $\angle B D F=\gamma^{\prime}, \angle B F D=\alpha^{\prime} ; \angle C E D=\alpha^{\prime}, \angle C D E=\beta^{\prime}$ ]. Then $\angle E A F=180^{\circ}-\left(\beta^{\prime}+\gamma^{\prime}\right)=\alpha$, and similarly $\angle F B D=\beta, \angle D C E=\gamma$. By symmetry it is enough to show that $\angle B A F=\alpha$ and $\angle A B F=\beta$ as well.

The perpendiculars from $F$ to $A E$ and $B D$ have the same length $s$. If the perpendicular from $F$ to $A B$ has length $h<s$, then $\angle B A F<\alpha$ and $\angle A B F<\beta$. If, on the other hand, $h>s$, then $\angle B A F>\alpha$ and $\angle A B F>\beta$. Since

$$
\angle B A F+\angle A B F=\alpha^{\prime}+\beta^{\prime}+60^{\circ}-180^{\circ}=\alpha+\beta,
$$

we see that necessarily $h=s$ and $\angle B A F=\alpha, \angle A B F=\beta$.

Yoshitake Hashimoto

Department of Mathematics

Graduate School of Science

Osaka City University

3-3-138, Sugimoto

Sumiyoshi-ku

Osaka, 558-8585 Japan

e-mail hashimot@sci.osaka-cu.ac.jp 